\title{
Erste Hilfe bei Gruppenarbeiten - Möglichkeiten der Studierenden zur erfolgreichen Gestaltung dieser Arbeitsform
}

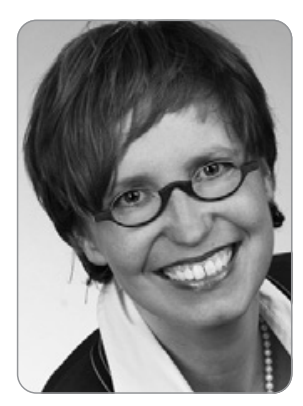

Prof. Dr. Juliane Wolf

ist Professorin für Betriebswirtschaft am Fachbereich Wirtschaft der Fachhochschule Münster. Bevorzugte Forschungsgebiete: Risikokultur, Führung, Coaching und Mentoring.

Stichwörter: Gruppenarbeit, Aktivierende Lehr- und Lernmethoden

1. Gruppenarbeiten als zunehmende Arbeitsform im Studium

Die an Hochschulen zunehmend eingesetzten aktivierenden Lern- und Lehrformate werden häufig als Gruppenarbeiten durchgeführt und das Ergebnis benotet. Damit sind das Funktionieren und die Leistungsfähigkeit der Gruppe entscheidend für den eigenen Studienerfolg. Es ist deshalb lohnenswert, sich damit zu beschäftigen, wie eine Gruppenarbeit erfolgreich gestaltet werden kann.

\section{Klärung der Rahmenbedingungen}

Die i. d. R. vom Dozenten vorgegebenen Rahmenbedingungen der Gruppenarbeit sind zu Beginn zu klären. Dies umfasst Aspekte wie Zeitraum der Bearbeitung, Form der Leistung (z. B. schriftliche Arbeit, Präsentation), Gruppenbildung sowie Bewertungsmaßstäbe (individuelle oder Gruppenbewertung) und Betreuungsmodalitäten (Häufigkeit und Inhalt von Besprechungen mit Dozenten, Verantwortlichkeit des Dozenten bei Schwierigkeiten im Gruppenprozess u. ̈̈.).

\section{Vorbereitung der Gruppenarbeit}

Es bietet sich an, dass die Teilnehmer zu Beginn nicht nur ein gemeinsames Verständnis der Zielsetzung entwickeln, sondern sich darüber hinaus auch über ihre individuellen Leistungsziele in Verbindung mit der Einsatzbe- reitschaft austauschen, um mögliche Diskrepanzen zwischen diesen Zielen und der Einsatzbereitschaft der Gruppenmitglieder zu erkennen. Eine geringe Anstrengungsbereitschaft kann darin begründet sein, dass die Arbeit für die Teilnehmer wenig bedeutungsvoll ist (vgl. z. B. Heckhausen 1977, Vroom 1964), weil z. B. die Note für sie ohne gravierende Relevanz ist oder sie die Sinnhaftigkeit der Aufgabe nicht sehen. Gelingt es nicht, hierfür zu begeistern, kann auch der Wunsch nach Zugehörigkeit zur Gruppe und die Identifikation mit der Gruppe dazu beitragen, dass Gruppenziele zu persönlichen Zielen werden und dadurch die Anstrengungsbereitschaft steigt.

Vielleicht zeigen sich mögliche Trittbrettfahrer schon in der Startphase. Trittbrettfahren basiert auf der bewussten Entscheidung eines Gruppenmitglieds, seine Leistung zu reduzieren (vgl. Kerr, 1983). Allerdings bestehen zwischen den Teilnehmern Informationsasymmetrien sowohl hinsichtlich der wahren Anstrengungsbereitschaft als auch der Leistungsfähigkeit und Trittbrettfahrer geben sich nicht zwangsläufig zu Beginn zu erkennen. Die erste Arbeitsphase kann dazu dienen, die Ernsthaftigkeit einer möglicherweise bekundeten Einsatzbereitschaft auch zu leben. Die ersten Arbeitspakete sollten daher eine überschaubare Größe haben und die ersten Treffen recht zeitnah geplant werden. So kann verhindert werden, dass Schwierigkeiten bei der Bearbeitung oder fehlende Ausarbeitungen erst kurz vor der Abgabe festgestellt werden.

Ein einheitliches Verständnis über die Zusammenarbeit kann auch durch das Aufstellen von gemeinsamen Spielregeln abgeglichen werden. Häufig finden Studierende das Aufstellen von Spielregeln zu formal und überflüssig. Allerdings gibt es nicht automatisch übereinstimmende Vorstellungen über die gemeinsame Zusammenarbeit. Ist es z. B. wirklich selbstverständlich, dass alle ihre Aufgaben zum vereinbarten Termin erledigen, wie Sie sich das vielleicht vorstellen? Ein frühes Aussprechen der eigenen Vorstellungen trägt dazu bei, Unterschiede schon beizeiten offenzulegen, einen Umgang damit zu finden und Konflikten vorzubeugen. 


\section{Arbeitsphase}

\subsection{Alternative Arbeitsaufteilungen und Konsequenzen für die Zusammenarbeit und Interaktion}

Zunächst ist zu klären, wie die Gruppe die Arbeit aufteilt. Manchmal erledigen Arbeitsgruppen (fast) alle Schritte gemeinsam. Bei überschaubaren Aufgaben mag dies durchführbar sein und hat den Vorteil, dass Teilnehmer Irrtümer Anderer korrigieren können, so dass daraus ein Lerneffekt sowie eine höhere Ergebnisqualität resultieren. Diese Vorgehensweise ist aufgrund der zahlreichen Mehrfacharbeiten jedoch recht ineffizient. Zudem lässt sich für den einzelnen Teilnehmer der eigene Leistungsbeitrag nicht mehr eindeutig identifizieren, was - anders als beim Trittbrettfahren durch eine bewusste Entscheidung - zu einem unbewussten Motivationsverlust führen kann (dem sog. "sozialen Faulenzen“; vgl. Williams/Harkins/Latane, 1981). Das Gruppenmitglied empfindet das eigene Anstrengungsniveau als angemessen, hat die Anstrengung aber tatsächlich reduziert. Allerdings führt eine erhöhte Anstrengung auch nur für einfache Aufgaben zu einer höheren Performance der Gruppe. Für komplexere Aufgabenstellungen, bei denen die Gruppenteilnehmer auch an den Aufgaben der anderen Teilnehmer arbeiten können, wird vermutet, dass mit einer höheren Anstrengung und der damit verbundenen Erregung die Fehlerhäufigkeit steigt und damit die Performance der Gruppenmitglieder sinkt. „Bei komplexen Aufgaben führt also gerade das soziale Faulenzen und die damit verbundene geringere Anstrengung sowie niedrigere Fehlerhäufigkeit $\mathrm{zu}$ einer höheren Leistung der Gruppe“ (vgl. Feuchter, 2001). Allerdings kann die Anwesenheit Anderer - auch ohne jegliches abwertendes Verhalten - bei komplexen Aufgaben zu sozialen Hemmnissen führen (vgl. Zajonic, 1965). Erleben sich leistungsschwache Teilnehmer zudem im ständigen Vergleich als unterlegen, kann dies den Glauben an die eigene Leistungsfähigkeit sowie die Bedeutung des Beitrags für das Gruppenergebnis reduzieren und damit die Motivation senken.

Alternativ kann die Gruppe Aufgaben aufteilen. Jedes Gruppenmitglied erarbeitet sich eigenständiges Wissen und macht sich für einen Teilbereich zum Experten. Um einerseits Verbindlichkeit zu schaffen und anderseits auch Missverständnisse $\mathrm{zu}$ verhindern, bietet sich eine schriftliche Dokumentation an, wer für welchen Bereiche als Experte verantwortlich ist. Bei der Arbeitsaufteilung ist der Beitrag des Einzelnen besser identifizierbar als bei der Variante „jeder macht alles", was die Motivation und Anstrengungsbereitschaft der Teilnehmer grundsätzlich erhöht und damit die Gefahr des sozialen Faulenzens reduziert (vgl. Williams/ Harkins/Latané, 1981). Zudem werden Doppelarbeiten vermieden und es können mehr Informationen erfasst und verarbeitet werden als durch Einzelarbeit oder die Vorgehensweise "jeder macht alles". Das gelingt jedoch nur, wenn die Gruppenmitglieder sich austauschen und ihr Wissen bewusst teilen (vgl. Brauner, 2003). Die Aufgaben sollten so aufgeteilt werden, dass sie zunächst unabhängig voneinander zu bearbeiten sind bzw. Abhängigkeiten in der Zeitplanung berücksichtigt werden und alle Teilnehmer herausfordernde, aber bewältigbare Aufgaben bearbeiten. In einer Gruppe, in der es für die Aufgabenverteilung aber keine eindeutige Verantwortlichkeit gibt, die sich der Problematik vielleicht auch nicht bewusst ist und darüber auch angesichts von Eigeninteressen der Teilnehmer Einigkeit erzielen muss, ist das eine sehr anspruchsvolle Aufgabe. Bei dieser Form der Arbeitsverteilung führt eine individuelle Bewertung der Leistung bei Trittbrettfahrern zumindest zum Teil dazu, dass sie die Konsequenzen ihres reduzierten Engagements tragen, bei einer Gruppennote profitiert der Trittbrettfahrer und die anderen Gruppenmitglieder sind von den negativen Auswirkungen wie Mehrarbeit und/oder schlechtere Note in vollem Ausmaß getroffen. Diese offensichtliche Ungerechtigkeit zu akzeptieren ist eine Herausforderung, um zu verhindern, dass die Teilnehmer nicht zum eigenen Schaden auch ihre Anstrengung bewusst zurückzufahren, um "nicht der Dumme zu sein“ (Kerr, 1983).

Gruppenarbeit bedeutet auch bei der Aufteilung der Arbeiten auf die Gruppenteilnehmer nicht, dass jedes Gruppenmitglied ab sofort nur individuell arbeitet und am Ende einfach alle Ergebnisse zusammengefügt werden. Damit der Vorteil des Wissens aller, der innerhalb einer Gruppe entstehenden Korrekturmöglichkeiten sowie die unterschiedlichen Blickwinkel genutzt werden können, ist die Koordination der Aktivitäten und die Interaktion der Teilnehmer erforderlich. So müssen die Teile konsistent aufeinander abgestimmt werden und mögliche inhaltliche Missverständnisse von der Gruppe durch kritisches Betrachten, Plausibilisieren u. Ä. korrigiert werden. Gruppen, die sich lediglich fertige Ergebnisse zuschicken, Diskussionen und Präsenzzeiten minimieren, nutzen die Vorteile nicht adäquat. Ergeben sich für Teilnehmer Schwierigkeiten bei der Bearbeitung, sollten sie das den anderen Gruppenmitgliedern kommunizieren, damit sich in der Gruppe die Bearbeitung anpassen lässt. Damit Teilnehmer bereit sind, ihre Schwächen offenzulegen, ist ein entsprechendes Vertrauensverhältnis erforderlich. Wünschenswert ist, dass leistungsstarke Teilnehmer einerseits eine Haltung entwickeln, in der sie schwächere Teilnehmer im Sinne des gemeinsamen Ziels kollegial unterstützen. Andererseits ist aber die Übernahme der Aufgaben durch die leistungsstärkeren Teilnehmer nur kurzfristig hilfreich, sondern sie sollten vielmehr bei Bedarf andere Teilnehmer an zentralen Punkten begleiten, ihrer Selbständigkeit fördern und er- 
mutigen statt zu entmutigen. Erfahren Teilnehmer Unterstützung von anderen Gruppenmitgliedern, so führt dies häufig dazu, dass sie künftig ihre Anstrengung für die Gruppe erhöhen (vgl. Hüffmeier et al., 2014), wird ihnen hingegen der Rhythmus der Überlegenen aufgezwungen, zerstört dies die Kooperation und die Leistung der schwächeren Teilnehmer reduziert sich weiter (vgl. Thomas, 1992).

\subsection{Und immer wieder bewusst gestaltete Interaktion als erforderliche Voraussetzung für ein gelungenes Gruppenergebnis}

Zur Ideensammlung wird auch von studentischen Gruppen manchmal ein Brainstorming durchgeführt. Intuitiv werden die Ideen häufig in der gesamten Gruppe zusammengetragen. Es hat sich jedoch gezeigt, dass ein solches Vorgehen - selbst wenn die Regeln des Brainstorming wie z. B. der Verzicht auf die sofortige Beurteilung von Ideen eingehalten werden - nicht zu den bestmöglichen Ergebnissen führt. So produzieren Gruppen quantitaiv weniger und qualitativ weniger gute Ideen als eine gleiche Anzahl von Einzelpersonen unabhängig voneinander. Ein maßgeblicher Grund liegt darin, dass während des Sprechens der Mitglieder die Ideenproduktion der anderen unterbrochen wird (vgl. Stroebe/Nijstad, 2004). Zielführender ist es daher, wenn zunächst jedes Gruppenmitglied einzeln seine Ideen sammelt und diese dann erst im Anschluss in der Gruppe zusammengetragen werden. Verlockend ist aber, dass die Beteiligung und Zufriedenheit der Teilnehmer höher ist, wenn ein Brainstorming in der Gruppe erfolgt (vgl. Zysno, 1998). Hier erfordert der Anspruch an die Qualität des Gruppenergebnisses ein Handeln wider die erste Intuition. Auch intensive Diskussionsprozesse sind für die Teilnehmer manchmal etwas lästig, allerdings sind sie erforderlich, um die bei Gruppen vorhandene Tendenz zur Angleichung von Meinungen und Verhalten (Konvergenztendenz) zu reduzieren. Abweichenden Meinungen wird in Gruppen zunächst viel Aufmerksamkeit geschenkt. Bei fehlender Beeinflussung kann es dazu kommen, dass das abweichende Gruppenmitglied ausgestoßen wird (vgl. Metz-Göckel, 2002). Es entsteht daher ein Konformitätsdruck und die Gefahr, dass die Gruppe nach Einmütigkeit strebt, was dazu beitragen kann, dass Gruppenentscheidungen schlechter ausfallen als die einzelner Personen (vgl. Janis, 1972). Auch Mehrheitsentscheidungen ohne angemessene vorherige Diskussion können verhindern, dass Gruppen gute Entscheidungen treffen. So können beispielsweise anderweitige, durchaus berechtigte Meinungen durch den Gruppendruck schnell abgewehrt werden oder alle glauben, die Anderen seien der gleichen Meinung, ohne dies jemals explizit $\mathrm{zu}$ erfragen. Solche Effekte treten unglücklicherweise gerade dann auf, wenn der Gruppenzusammenhalt (Kohäsion) besonders hoch ist (vgl. Schulz-Hardt, 1997), was unter Motivations- und Zufriedenheitsaspekten für die Gruppen wünschenswert ist. Für ein inhaltlich gutes Ergebnis ist aber wichtig, dass das gesamte in der Gruppe vorhandene Wissen genutzt wird. Zumindest an zentralen Punkten ist es daher wichtig, immer wieder zu erfragen, ob es in der Gruppe noch weitere Ansichten gibt und die Argumente hierzu hinreichend auszutauschen und im weiteren Prozess zu berücksichtigen.

Vorhandene Erkenntnisse zu nutzen, bedeutet auch, dass die Teilnehmer sich z. B. im Rahmen einer anstehenden Präsentation ihre Teile gegenseitig vortragen und dazu einander Feedback geben.

Es hat sich gezeigt, dass eine erfolgreiche Gruppenarbeit durch vielfältige Kommunikationsprozesse gekennzeichnet ist. In einem Team würden solche Prozesse häufig von einem Moderator gestaltet. Eine Arbeitsgruppe könnte sich die Aufgaben eines Moderator (Zeitmanagement, alle Teilnehmer zu Wort kommen lassen, Positionen auf den Punkt bringen, Herausgearbeitetes zusammenfassen und dokumentieren) abwechselnd an einen Teilnehmer übertragen oder alternativ auch die Aufgaben aufteilen und auf verschiedene Teilnehmer verteilen. Ein Diskussionsleiter, der die Verantwortung dafür hat, die Mitglieder zu ermuntern, ihre Meinung zum Ausdruck zu bringen und zu begründen, kann die Ergebnisqualität nachweislich verbessern (vgl. Maier/Solem, 1952).

\section{Umgang mit schwierigen Situationen/Konflikten}

Eine gute Gruppenarbeit zeichnet sich nicht dadurch aus, dass sie stets vollkommen harmonisch ist. Vielmehr wurde ja schon aufgezeigt, dass das Bestreben nach Einmütigkeit sogar die Qualität des Gruppenergebnisses beeinträchtigen kann. Zudem ist es typisch, dass im Rahmen der Phasen, die Gruppen durchlaufen (vgl. Tuckmann, 1965) nach anfänglicher Harmonie die Gruppen in eine sogenannte Storming-Phase geraten, wenn die Gruppenmitglieder feststellen, dass sie unterschiedliche Persönlichkeiten und Vorstellungen haben. Entscheidend für eine weitere konstruktive Zusammenarbeit ist der adäquate Umgang mit den sich daraus ergebenden Konflikten. Dazu gehört, diese frühzeitig anzusprechen, denn schwelende Konflikte eskalieren typischerweise und lassen sich ab einem bestimmten Stadium nicht mehr durch die Beteiligten lösen. Dabei sollte die Beobachtung möglichst konkret benannt und die störende Wirkung für den Betroffenen beschrieben werden. Auch ist die Sichtweise des Gesprächspartners zu erfragen und ernsthaft nachzuvollziehen, wie sich das aus dessen Sicht darstellen könnte. Konflikte entstehen nicht zwangsläufig, weil einer „falsch“ ist oder sich falsch verhält, son- 
dern können auch mit unseren eigenen Sichtweisen zusammenhängen oder dem Zusammenspiel zwischen den Beteiligten. Ziel ist es, möglichst konkrete Vereinbarungen über den künftigen Umgang mit den Schwierigkeiten zu finden, die ggf. auch in die Spielregeln aufgenommen werden können. Sollten die Teilnehmer nach intensivem Versuch keine Lösung finden, so ist der Dozent zur Klärung mit einzubinden, sofern er die Bereitschaft dazu signalisiert hat.

\section{Abschluss der Gruppenarbeit}

Lernprozesse finden insbesondere dann statt, wenn erfolgreiche und nicht erfolgreiche Verhaltensweisen erkannt und ggfs. alternative Handlungsweisen entwickelt werden. Um die Fähigkeit zur Arbeit in einer Gruppe weiterzuentwickeln, ist daher - neben der inhaltlichen Betrachtung eine Reflexion über Stärken und Schwächen mit gegenseitigen Rückmeldungen im Gruppenprozess hilfreich.

\section{Literatur}

Brauner, E., Informationsverarbeitung in Gruppen: Transaktive Wissenssysteme, in: Stumpf, A., Thomas, A. (Hrsg.), Teamarbeit und Teamentwicklung, Göttingen 2003, S. 57-83.

Feuchter, A.. Lob des sozialen Faulenzens. Motivation und Leistung beim Lösen komplexer Probleme in sozialen Situationen. Lengerich 2001.

Hüffmeier, J., Wessolowski, K, van Randenborgh, A., Bothin, J. Schmid-Loertzer, N., Hertel, G., Social support from fellow group members triggers addition effort in groups, in: European Journal of Social Psychologie, 44. Jg. (2014), S. 287-296.

Janis, I. L., Victims of groupthink, Boston 1972.

Kerr, N.L., Motivation Losses in small groups: A social dilemma analyses, in: Journal of Personality and Social Psychologie, 45. Jg. (1983), S. 819828.

Maier, N.R.F., Solem, A.R., The contribution of a discussion leader to the quality of group thinking: The effective use of minority opinion, in: $\mathrm{Hu}-$ man Relations, 5. Jg. (1952), S. 277-288.

Metz-Göeckel, H., Psychologie der Gruppe, http://eldorado.tu-dortmund. de/bitstream/2003/2953/1/Psychologie.pdf (Abrufdatum: 24.03.2018).

Schulz-Hardt, S., Realitätsflucht in Entscheidungsprozessen. Vom Groupthink zum Entscheidungsautismus, Bern 1997.

Stroebe, W., Nijstad, B., Warum Brainstorming in Gruppen Kreativität vermindert: Eine kognitive Theorie der Leistungsverluste beim Brainstorming, in: Psychologische Rundschau, 55. Jg. (2004), S. 2-10.

Thomas, A., Grundriß der Sozialpsychologie, Bd. 2: Individuum - Gruppe Gesellschaft, Göttingen 1992.

Tuckman, I. W., Developmental sequence in small groups, in: Psychological Bulletin, 63. Jg. (1965), S. 384-399.

Vroom, V.H., Work and motivation. New York 1964.

Williams, K., Harkins, S.G., Lantané, B., Identifiability as a determinant to social loafing: Two cheering experiments, in: Journal of Personality and Social Psychology, 40. Jg. (1981), S. 303-311.

Zajonic, R. B., Social faciliation, in: Science, 149. Jg. (1965), S. 269-274. Zysno, P., Von Seilzug bis Brainstorming: Die Effizienz der Gruppe, in: Witte, E.H. (Hrsg.), Sozialpsychologie der Gruppenleistung, Lengerich 1998, S. $184-210$.

\section{Unternehmen der Zukunft.}

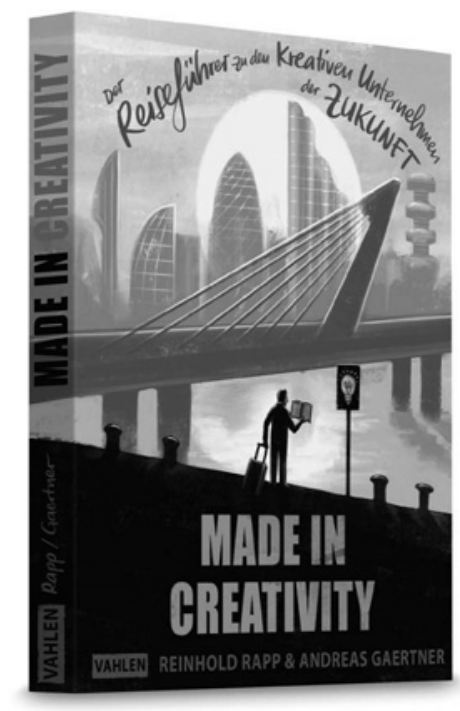

Rapp/Gaertner

Made in Creativity

Der Reiseführer zu den kreativen Unternehmen der Zukunft Mit einem Vorwort von Prof. Dr. Hermann Simon und Matthias Horx

2019. 239 Seiten. Klappenbroschur $€ 29,80$

ISBN 978-3-8006-5954-8

\section{Der neue "Reiseführer"}

ist wunderbar illustriert und zeigt,

- wie die Kreativität von Teams die Künstliche Intelligenz von Maschinen auf allen Gestaltungsfeldern übertrumpfen kann,

- wie ein Zusammenklang zwischen nachhaltigen Unternehmensformen und neuen, noch in der Entwicklung befindlichen Typen Dynamik und Substanz erzeugt und

- wie durch Integration von Kunden und Partnern ein permanentes Co-Creating zu Wettbewerbsvorteilen wird.

\section{Ein Buch}

für alle, die kreativ etwas Neues in ihren Organisationen schaffen wollen.

Erhältlich im Buchhandel oder bei vahlen.de | Verlag Franz Vahlen GmbH · 80791 München kundenservice@beck.de | Preise inkl. MwSt. | 170842

\section{Vahlen}

